\title{
Web Service para previsão de tempo e clima através de dados georeferenciados
}

\author{
João Paulo Moreira Prestes ${ }^{1}$ \\ Carlos Amaral Hölbig ${ }^{1}$ \\ Willingthon Pavan ${ }^{1}$ \\ José Maurício Cunha Fernandes ${ }^{2}$
}

\begin{abstract}
Resumo: Atualmente o Centro de Previsão de Tempo e Estudos Climáticos (CPTEC) disponibiliza gratuitamente a sociedade informações de previsões de tempo e clima para todo território brasileiro, entretanto, apenas uma restrita parcela da sociedade possui conhecimento suficiente para a consulta e extração destas informações. Desta forma, este trabalho tem como objetivo apresentar uma estrutura computacional capaz de fornecer informações de previsões de tempo e clima, através do uso de tecnologias e dados georeferenciados. Os testes, protótipos e validações deixam claros as funcionalidades, viabilidade e usabilidade da solução.
\end{abstract}

Palavras-chave: Previsão de tempo, Web Services, GrADS.

\begin{abstract}
The CPTEC, Centro de Previsão de Tempo e Estudos Climáticos, provides information about forecast for all Brazilian territory, however, only restricted part of the society has enough knowledge to get and manipulate these kind of data. Therefore, this paper has as goal to present a computational structure capable to supply information about forecasts, through the use of technologies and georeferentials data. The tests, prototypes and validations show the functionalities, viability and usability of this solution.
\end{abstract}

Keywords: Forecast, Web Services, GrADS

\section{Introdução}

A meteorologia atingiu ao longo dos tempos um nível muito sofisticado com base na importância prática da previsão do tempo e clima, tornando-se uma ciência muito desenvolvida, sendo as informações meteorológicas, atualmente, vitais para a sobrevivência humana. A previsão de nebulosidade, visibilidade e condições do vento nos aeroportos, por exemplo, são necessárias para planejar a decolagem e a aterrisagem, sendo informações valiosas, também, para determinar a quantidade de combustível necessário na determinação de uma rota. A previsão do tempo para a navegação marítima também se faz importante para que a tripulação e a carga cheguem ao seu destino. Previsões de tempestades, chuvas, ventos e formações de geleiras são indispensáveis, auxiliando na determinação de uma melhor rota.

O tempo e o clima também interferem na indústria, no comércio e na agricultura, no que tange ao transporte de cargas, na execução de serviços, na previsão de consumo e épocas de plantio e colheita [18]. O órgão nacional responsável pelas informações de previsões de tempo e clima no Brasil é o Instituto Nacional de Pesquisas Espaciais (INPE), através do Centro de Previsão de Tempo e Estudos Climáticos (CPTEC), o qual

\footnotetext{
${ }^{1}$ Curso de Ciência da Computação, UPF, Campus 1 - BR 285 - Passo Fundo (RS) - Brasil

jpprestes@gmail.com, holbig@upf.br, pavan@upf.br

${ }^{2}$ Embrapa Trigo, Rodovia BR 285, km 294 - Passo Fundo (RS) - Brasil

mauricio@cnpt.embrapa.br
}

http://dx.doi.org/10.5335/rbca.2011.1371 
executa periodicamente diversos modelos numéricos de previsão de tempo. Um dos modelos mais conhecidos e difundidos é o modelo ETA, um modelo de mesoescala, em ponto de grade e equações primitivas. A letra grega ETA $(\eta)$ dá o nome ao modelo, sendo a topografia representada em forma de degraus. A versão deste modelo é hidrostática e cobre a maior parte da América do Sul e oceanos adjacentes.

A resolução horizontal do modelo ETA é de $40 \mathrm{~km}$ e a vertical de 38 camadas. As informações com as previsões de tempo são fornecidas duas vezes ao dia, uma com condição inicial as 00 e outra às 12 UTC [5]. Os resultados deste modelo são disponibilizados gratuitamente para a sociedade através da internet, porém, uma grande parcela da população não possui infra-estrutura e conhecimentos necessários para manipular e interpretar os resultados destes modelos.

Os arquivos disponibilizados pelo CPTEC estão em um formato baseado na linguagem de scripts chamado GrADS (Grid Display Analisys System). O GrADS é essencialmente um software para visualização e análise de dados em pontos de grade. Tratando-se de visualização, este é o software mais utilizado na área de pesquisa e meteorologia em todo o mundo. Uma das principais vantagens do GrADS é a sua distribuição gratuita pela internet para os diversos tipos de ambientes, podendo ser implementado em diversos sistemas operacionais, tais como UNIX, LINUX e DOS [3].

Com o objetivo de facilitar o acesso às informações de previsão de tempo e clima, este trabalho tem como objetivo apresentar uma estrutura computacional capaz de fornecer previsões de tempo e clima, extraídas dos arquivos disponibilizados pelo CPTEC, através de um sistema de web service. Para teste e validação da solução desenvolvida foi criado uma aplicação cliente, a qual utiliza os recursos das bibliotecas do Google Maps para fazer solicitações ao sistema de web service, através das coordenadas de latitude e longitude extraídas dos mapas. O desenvolvimento deste trabalho abrangeu estudos bibliográficos e pesquisas na internet, com o objetivo de extrair o máximo de informações necessárias para que a aplicação alcançasse todos seus objetivos.

\section{Revisão de Literatura}

\subsection{Meteorologia}

A meteorologia proveniente das palavras gregas meteoros (elevado no ar) e logos (estudo) é a ciência que estuda os processos que ocorrem na atmosfera terrestre com aproximadamente $20 \mathrm{~km}$ de espessura. Devido ao fato da maioria das atividades humanas ocorrerem nesta camada é necessário estudar e compreender melhor os processos que causam a evolução das condições do tempo [8]. Os aspectos mais difundidos na Meteorologia são: a previsão do templo e a climatologia. O tempo pode ser definido como o estado da atmosfera em determinado instante e lugar. O clima tem sido frequentemente definido como um conjunto de condições normais que dominam uma região, obtidas das médias das observações em um determinado intervalo de tempo [8].

\subsection{Previsão de tempo e clima}

$\mathrm{Na}$ maioria dos casos as definições de tempo e clima são confundidas. O tempo diz respeito à situação atual da nossa atmosfera, enquanto o clima representa um longo período a respeito do comportamento do tempo. Em outras palavras, o tempo meteorológico é o estado instantâneo da atmosfera e o clima corresponde a uma integração de condições do tempo para um determinado período de tempo em uma determinada área geográfica [6]. A previsão do tempo é probabilística, ou seja, sugere a probabilidade de ocorrência de determinados fenômenos meteorológicos. A previsão do tempo é um prognóstico das condições atmosféricas que poderão ou não ocorrer para os próximos minutos, horas ou dias adiante [6].

A previsão de clima tem por finalidade prever, por exemplo, a qualidade da estação chuvosa como um todo, numa determinada região, num determinado período de tempo. Embora a previsão climática também seja probabilística, esta em alguns casos pode ser mais precisa que a previsão do tempo [6]. Portanto, a previsão meteorológica procura como um todo antecipar probabilisticamente o que irá acontecer na atmosfera um determinado período de tempo para uma determinada região. Embora a mesma não apresente uma antevisão exata das condições do tempo e clima, isso não diminui a importância econômica e social que ela representa [6].

Revista Brasileira de Computação Aplicada (ISSN 2176-6649), Passo Fundo, v.3, n. 2, p. 2-16, set. 2011 


\subsection{Fenômenos meteorológicos}

A radiação solar é a principal fonte de energia da superfície de nosso planeta. São as variações de intensidade da radiação recebida, decorrentes dos movimentos da terra, que provocam a maior parte das modificações nas condições atmosféricas, resultando em fenômenos como a chuva, o vento e outros mais. Os dados resultantes de um modelo numérico são denominados de mapas de prognóstico, os quais podem conter diversas informações, tais como: temperatura, umidade, cobertura das nuvens, pressão atmosférica, vento, precipitação, descargas elétricas, nevoeiro, orvalho, geada, entre outros [16].

\subsection{Modelos de Previsão do Tempo e Clima}

Os modelos de previsão numéricos são constituídos de modelos computacionais os quais formam a base para a previsão de tempo e clima no mundo. Os Modelos de Circulação Geral (MCGs) apresentam bons índices de acerto e representação das previsões de tempo e clima. Entretanto há uma limitação para a representação dos processos de mesoescala devido à baixa resolução apresentada por estes MCG’s [2].

\subsection{Classificação dos modelos}

Os modelos de previsão numérica do tempo estão divididos em duas categorias, conforme sua escala espacial dentro da qual a previsão é realizada: Global, com resolução da ordem de $200 \mathrm{~km}$, a qual visa identificar o comportamento geral da atmosfera sobre uma área extensa, sendo estes modelos capazes apenas de identificar fenômenos meteorológicos de larga escala (ditos sinópticos) [2]; e Regional ou local, com resolução espacial de alguns metros a cerca de $50 \mathrm{~km}$. Visa identificar com maior detalhe o comportamento da atmosfera sobre uma região específica, sendo capazes de identificar fenômenos meteorológicos de pequena escala (ditos de mesoescala) [2];

\subsection{Modelo Regional ETA}

O modelo ETA é um modelo de mesoescala, em ponto de grade, de equações primitivas. A versão do modelo Eta que roda operacionalmente no CPTEC é hidrostático e cobre a maior parte da América do Sul e oceanos adjacentes [5]. A resolução horizontal atual é de $40 \mathrm{~km}$ e a vertical de 38 camadas. As previsões são fornecidas duas vezes ao dia, uma com condição inicial às 00 e outra às 12 UTC. A condição inicial é proveniente da análise do National Centers for Environmental Prediction (NCEP) e as condições de contorno lateral são provenientes das previsões do modelo global do CPTEC e atualizada a cada 6 horas. O prazo de integração é de 72 horas [5]. A letra grega eta $(\eta)$ dá o nome ao modelo, sendo a topografia é representada em forma de degraus, proporcionando as variáveis prognosticas [5].

\section{Material e Métodos}

\subsection{GrADS}

Para extrair as informações das previsões de tempo e clima gerados pelo modelo numérico do CPTEC, foi utilizado o software GrADS (Grid Analysis and Display System). Esta ferramenta é utilizada para visualizar e analisar dados de previsão de tempo em ponto de grade. Atualmente é a ferramenta mais difundida e utilizada pelos Centros de Pesquisas Especiais em todo o mundo. O GrADS trabalha com quatro dimensões: latitude, longitude, nível e tempo [3]. Na maioria das vezes os dados com as previsões do tempo são armazenados em dois arquivos: arquivo binário (grb), contendo as quatro dimensões bem como os valores das previsões; e arquivo descritor (ctl), o qual contém o índice dos dados armazenados no arquivo binário. Diversas são as vantagens do GrADS, por exemplo, a distribuição gratuita através da internet, bem como a possibilidade de execução na maioria dos sistemas operacionais utilizados no mundo [3]. 


\subsection{Java}

No desenvolvimento do sistema de Web service foi utilizada a linguagem de programação Java. Esta é uma tecnologia computacional desenvolvida pela Sun Microsystems e lançada em 1995. A plataforma Java dispõe de diversos recursos para o desenvolvimento de aplicações, sejam elas destinadas a desktops, dispositivas móveis ou mesmo aplicações distribuídas [9]. Atualmente a tecnologia dispõe de três pacotes de API's: J2SE ferramentas e API's essenciais para o desenvolvimento da maioria das aplicações Java. Útil para o desenvolvimento de aplicações para desktop com ou sem interfaces gráficas; J2EE - ferramentas e API's destinadas ao desenvolvimento de aplicações distribuídas, através de algumas tecnologias como: RMI, EJB, CORBA, JMS, etc; e J2ME - ferramentas e API's para o desenvolvimento de aplicações para dispositivos portáteis (palms, celulares, eletrodomésticos, entre outros);

A linguagem de programação Java é altamente portável, devido a sua interpretação através da Java Virtual Machine (JVM) e um código semi-compilado denominado bytecodes. Os bytecodes podem ser carregados dinamicamente pela JVM a qualquer momento dependendo do fluxo de execução da aplicação. O gerenciamento automático da memória e a possibilidade de utilizar recursos multithreading são outras vantagens substancias para solidificar a tecnologia Java como uma ótima opção para o desenvolvimento de sistemas distribuídos [9].

\subsection{PHP}

Para a cópia e sincronização dos arquivos disponibilizados pelo CPTEC com o servidor de aplicação, foi utilizada a linguagem de programação PHP. O PHP (acrônimo para PHP: Hypertext Preprocessor) é uma linguagem de programação interpretada, muito utilizada para o desenvolvimento de websites dinâmicos, graças sua fácil integração com HTML. É uma linguagem server-side, ou seja, executa no servidor e retorna o resultado da requisição via HTTP, quando utilizada em conjunto com um servidor de web, como por exemplo o Apache. PHP é uma linguagem de fácil aprendizado pelo fato de sua similaridade com as linguagens C, Java e Perl. A versão 5.0 da linguagem oferece diversas funcionalidades, destacando-se, dentre elas, a característica para o desenvolvimento de aplicações utilizando o paradigma de orientação a objetos. Embora a maioria dos programadores PHP utilize a linguagem para o desenvolvimento de aplicações para internet, é possível também desenvolver scripts que executem determinadas rotinas no próprio servidor, como por exemplo, rotinas de manutenção do servidor, backups, etc. Com relação à conectividade com banco de dados, o PHP oferece possibilidade de acesso à maioria dos disponíveis no mercado, como: Oracle, Sybase, Mysql, Postgres, dentre outras formas conexões ODBC [11].

\subsection{Web service}

Com o objetivo de facilitar o acesso aos dados da previsão de tempo e manter uma interoperabilidade da solução, foi optado pelo desenvolvimento um Web Service para a disponibilização das informações. web service é um tipo de aplicação distribuída muito utilizada para a integração de sistemas, tendo em vista que esta é independente de plataforma [19]. São componentes baseados em padrões (definidos pela W3C) de comunicação bem difundidos e amplamente utilizados. Sua forma de comunicação e troca de dados acontece através da internet com arquivos no formato XML (eXtensible Markup Language). As chamadas as operações, incluindo parâmetros de entrada e saída são codificados através do protocolo denominado SOAP (Simple Object Access Protocol). As operações, mensagens e parâmetros de comunicação são descritos através da linguagem WSDL (Web Services Definition Language). A publicação, pesquisa e descoberta dos serviços são realizadas através do protocolo UDDI (Universal Description, Discovery and Integration). Através destas tecnologias é possível construir sistemas distribuídos, com soluções integradas, independente de sistema e plataforma utilizada [14,19].

\subsection{Apache Tomcat}

Para a disponibilização do web service na internet, foi utilizado o servidor de aplicações Java Apache Tomcat, ou simplesmente Tomcat, como é conhecido. O Tomcat foi desenvolvido dentro do projeto Jakarta (projeto de desenvolvimento de diversos softwares livres em Java) pela Apache Software Foundation com apoio da Sun Microsystems, possuindo, atualmente, seu próprio projeto. Dentre as diversas vantagens deste servidor de aplicações podemos destacar a robustez e eficiência, mesmo utilizado em ambientes de produção críticos. O 
Tomcat é um container web, o qual referencia grande parte da especificação J2EE da tecnologia Java, através dos recursos Servlet e JSP, além das tecnologias JNDI e JDBC. Além de servidor de aplicações, o Tomcat também oferece recurso para servidor Web/HTTP [15].

\subsection{Apache Axis}

Após o desenvolvimento do web service, foi optado pela utilização deste serviço através do framework de código aberto denominado Apache Axis. Este framework foi desenvolvido com base na linguagem Java, utilizando XML e o protocolo SOAP [1,12]. O Apache Axis permite que programadores desenvolvam aplicações distribuídas através da linguagem Java, sendo possível, também, a implementação utilizando a linguagem C++. $\mathrm{O}$ framework Axis foi desenvolvido pela Apache Software Foundation, mesma empresa que mantém atualmente a ferramenta. Utilizando o Axis é possível publicar os serviços através de duas formas: arquivo JWS (Java WebService) ou então através da publicação da WSDD (Web Service Deployment Descriptor) a qual descreve com detalhes como serão criados os serviços a partir das classes Java já existentes. Para o funcionamento do Axis, basta disponibilizar um servlet do framework em um servidor Apache Tomcat. Na Figura 1 é possível visualizar o funcionamento completo do Apache Axis.

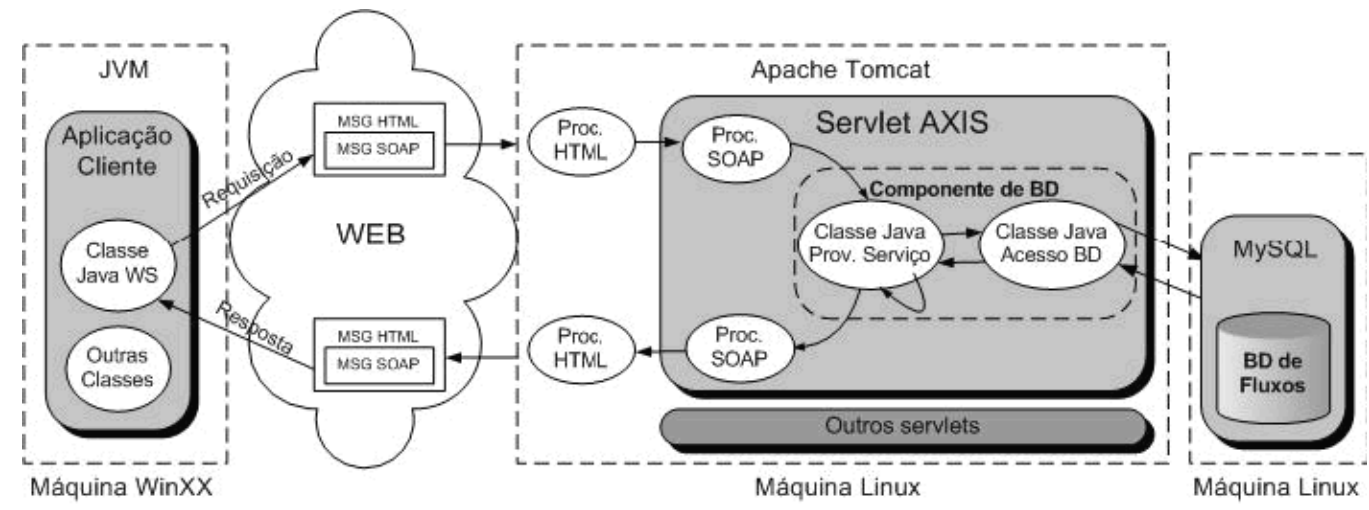

Figura 1. Estrutura de funcionamento do Apache Axis. Fonte: [1]

\subsection{Google Maps}

Com o objetivo de validar a solução proposta, foram utilizadas as bibliotecas de mapas do Google Maps. O Google Maps é um serviço de pesquisa e visualização de mapas com fotos de satélite desenvolvido pela empresa Google. É possível utilizar as bibliotecas e API’s desenvolvidas em Javascript em sua própria página da internet [10].

O mapa pode ser consultado usando texto livre, permitindo drill-down para especificar endereços de ruas e locais, como hotéis e restaurantes. As funções de busca são como em uma aplicação web clássica, atualizando a página inteira, mas o mapa em si é enriquecido com AJAX (Asynchronous Javascript And XML). Clicando nos links individuais de uma busca por hotéis, por exemplo, mostrará informações adicionais flutuando sobre o local do mapa relacionado, e até mesmo quando o mapa é transladado, o balão com as informações é ajustado. A transladação do mapa em si é a característica mais interessante do Google Maps. O usuário pode arrastar o mapa inteiro utilizando apenas o mouse. O mapa em si é composto de pequenos pedaços de imagens, e se o usuário arrastar o mapa além da parte exposta, as imagens relacionadas serão assincronamente carregas [7].

O Google disponibiliza para os desenvolvedores uma API (Application Program Interface) de forma que estes possam criar seus próprios serviços utilizando os recursos do Google Maps, ou seja, é possível adicionar recursos de visualização de mapas dentro do seu próprio website de maneira muito simples. A API do Google Maps encontra-se na segunda versão com diversas funcionalidades. Utilizando esta API é possível adicionar um mapa em um website e com um simples clique do mouse sobre a região desejada é possível, por exemplo, extrair informações georeferenciadas sobre o ponto clicado [7]. 


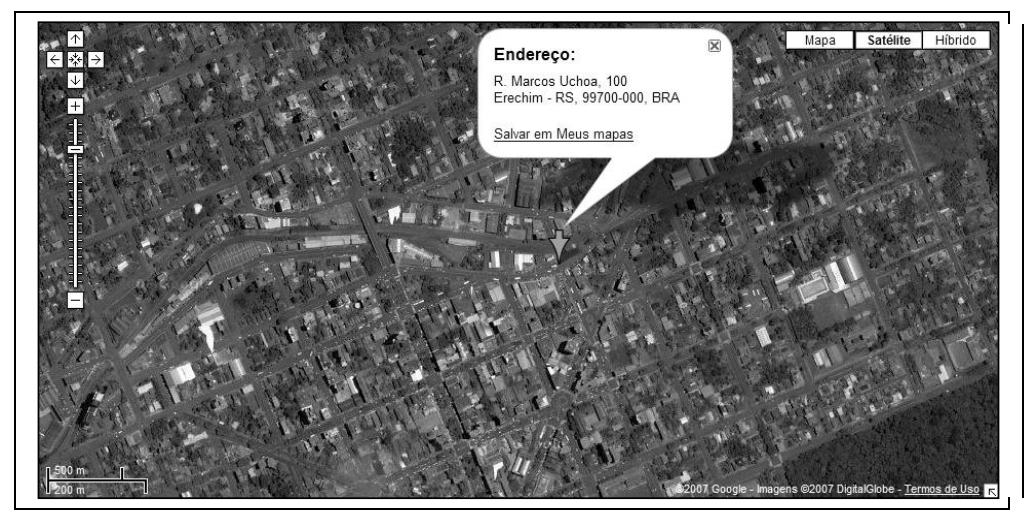

Figura 2. Imagem capturada através do Google Maps.

\subsection{Ubuntu Linux}

O sistema operacional utilizado para o desenvolvimento e implantação da solução foi o Ubuntu/Linux. O Ubuntu é um sistema operacional baseado na distribuição Debian. É patrocinado pela Canonical Ltda e o seu nome deriva do conceito sul africano Ubuntu diretamente traduzido como "humanidade para com os outros". Diferencia-se do Debian por ser lançado semestralmente, por disponibilizar suporte técnico nos dezoito meses seguintes ao lançamento de cada versão e pela filosofia em torno de sua concepção, uso e distribuição[17]. A proposta do Ubuntu é oferecer um sistema operacional que qualquer pessoa possa utilizar sem dificuldades, independente de nacionalidade, nível de conhecimento ou limitações físicas. A distribuição deve ser constituída totalmente de software gratuito e livre, além de isenta de qualquer.

\section{Resultados}

\subsection{Identificação do problema}

Atualmente o CPTEC disponibiliza gratuitamente para a sociedade, através da internet, dados referente a previsões de tempo e clima, extraídos diariamente de seu modelo numérico regional, denominado ETA. Estas informações são disponibilizadas em dois formatos de arquivos, um com a extensão "grb" e outro com a extensão "ctl". O arquivo "ctl" funciona como índice para os dados contidos no arquivo binário "grb". Sempre que necessário extrair ou consultar uma informação a respeito das previsões de tempo e clima contidos nestes arquivos, é necessário utilizar um software denominado GrADS, utilizado por grande parte dos centros de pesquisas espaciais do mundo [5].

Embora os métodos e ferramentas utilizados pelo CPTEC para a disponibilização destas informações sejam muito difundidas na área da meteorologia, estas informações possuem uma estrutura e forma de acesso desconhecida da maioria dos usuários que necessitam destas informações. A instalação e configuração software GrADS, para a aquisição de tais informações pode se tornar custosa para usuários leigos que não dominam a utilização de computadores, mas possuem a necessidade de acesso as informações. Tendo esta vista esta dificuldade de acesso, buscou-se o desenvolvimento de uma interface, através de tecnologias difundidas, de forma que facilite o acesso aos dados de previsão de tempo e clima.

\subsection{Objetivo da solução proposta}

Desenvolver uma estrutura computacional capaz de fornecer informações de previsão de tempo através do uso de tecnologias e dados georeferenciados. O desenvolvimento desta estrutura foi realizado através da criação de um web service capaz de fornecer informações de previsão do tempo e clima de maneira amigável e legível para qualquer tipo de usuários e/ou sistema. 


\subsection{Estrutura computacional}

Para a disponibilização do serviço, foi necessária a criação de uma estrutura computacional, conforme ilustrada na Figura 3:

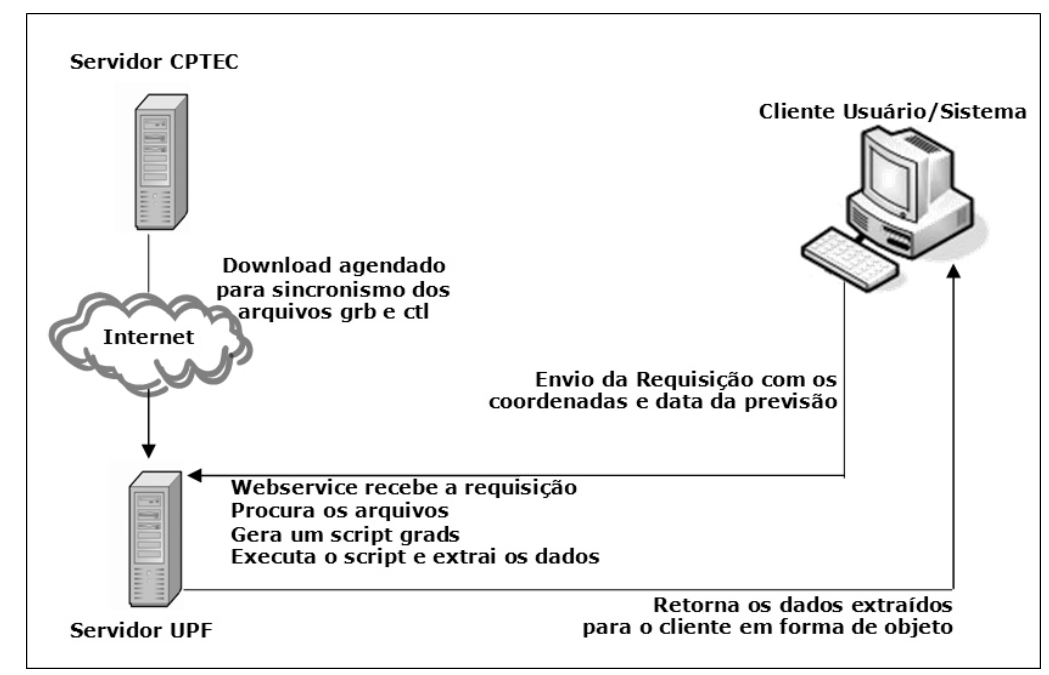

Figura 3. Estrutura computacional da solução

\subsection{Sincronismo dos dados}

Conforme citado anteriormente, o CPTEC disponibiliza diariamente arquivos com as previsões de tempo e clima para todo o território brasileiro. Tais arquivos podem ser obtidos através do endereço $\mathrm{ftp}: / / \mathrm{ftp} 1 . c p t e c$. inpe.br/modelos/io/tempo/regional/Eta40km/regions/. Estes arquivos estão divididos em diretórios conforme as regiões brasileiras e são disponibilizados duas vezes ao dia, um as 00 hora UTC e outra às 12 horas UTC. Cada publicação dos arquivos na internet possui previsões para os cinco dias subseqüentes, em um intervalo de seis em seis horas, ou seja, as $00,06,12$ e 18 horas de cada dia. Com o objetivo de agilizar o processo de consulta e extração dos dados de tempo e clima, foi optado por realizar uma cópia dos arquivos disponíveis nos servidores do CPTEC, através de um script desenvolvido com a linguagem de scripts PHP (Figura 4).

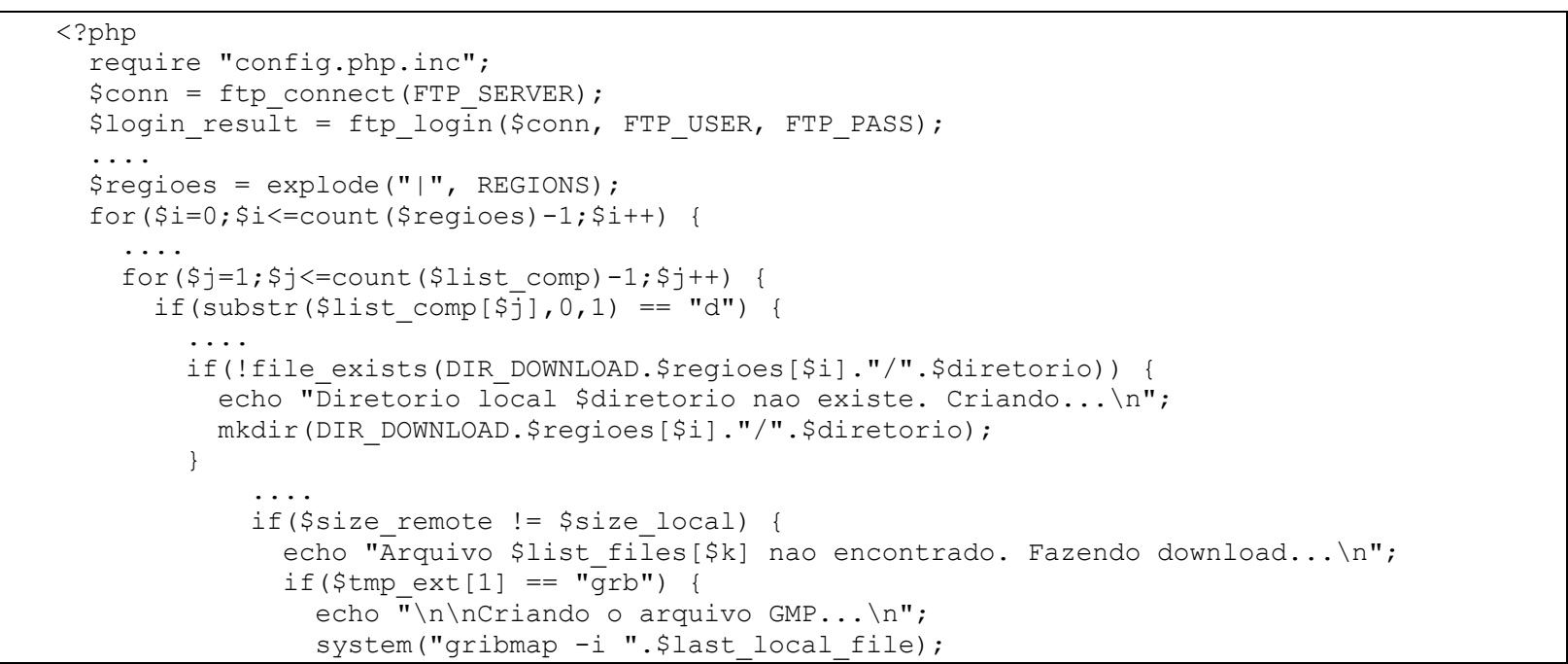

Figura 4. Partes do script para sincronismo dos arquivos do CPTEC.

Este script tem como objetivo procurar todos os arquivos que não existem na base de dados local e copiálos automaticamente, mantendo assim uma base de dados sincronizada com o CPTEC. Este script está agendado 
para executar de uma em uma hora, através do sistema de agendamento de tarefas do sistema operacional Linux, denominado "Cron" [4].

A cada execução do script, o mesmo verifica o tamanho de cada arquivo (remoto e local). Caso o arquivo esteja incompleto, o download é iniciado novamente para garantir a integridade dos dados. Caso os arquivos tenham o mesmo tamanho, este é ignorado e verificado o próximo. Na Figura 5 é apresentado o arquivo de log gerado pelo script:

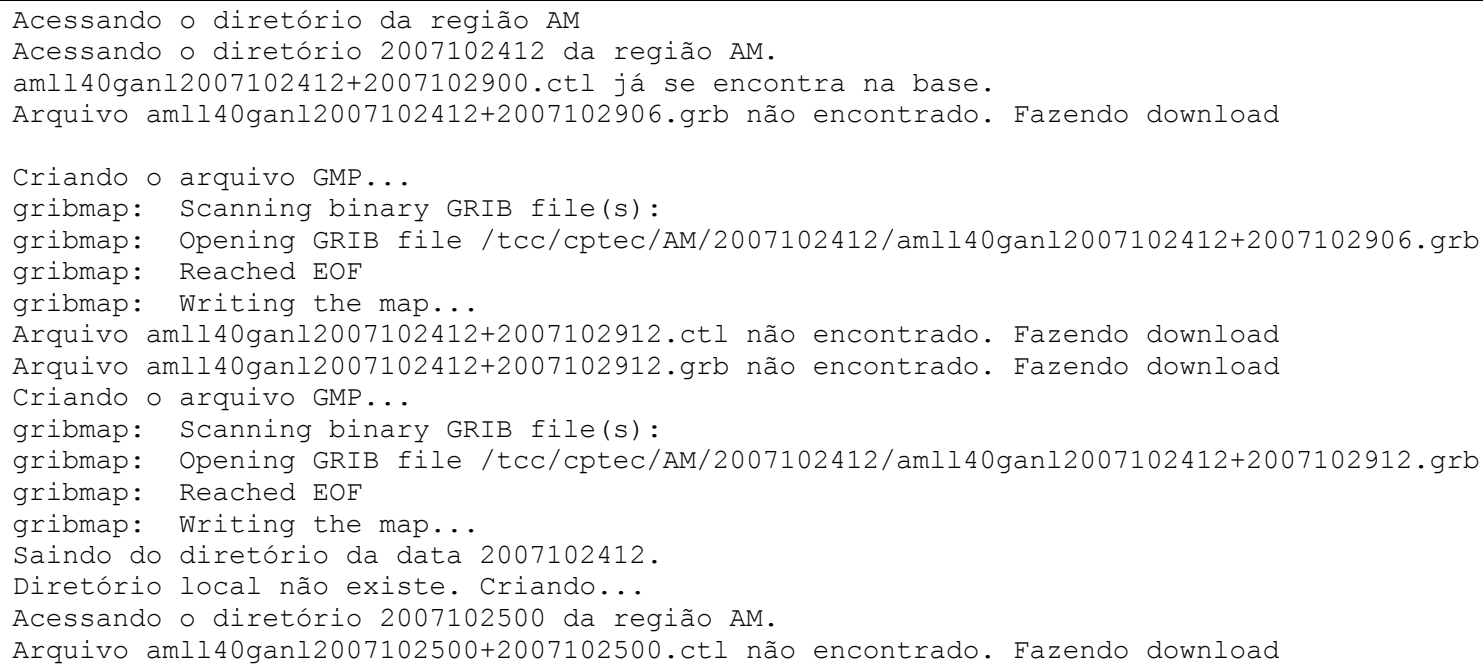

Figura 5. Arquivo de log do script de sincronização

Devido ao fato do CPTEC não disponibilizar o arquivo com o índice dos dados binários, foi necessário realizar, após a sincronização dos arquivos "ctl" e "grb", a criação deste arquivo através do comando gribmap do próprio GrADS, conforme exemplo:

$$
\text { joaopaulo@ubuntu:/> gribmap -i arquivo_descritor.ctl }
$$

\subsection{Desenvolvimento do Web Service}

Com o objetivo de facilitar o acesso às informações de previsão de tempo e clima, foi desenvolvida uma aplicação utilizando a tecnologia de web services, tendo em vista a intenção de que a comunicação entre aplicações seja realizada através da internet de forma independente de sistema operacional e linguagem de programação, mantendo assim a interoperabilidade da solução. O desenvolvimento do Web Service foi realizado através da linguagem de programação Java, visto que esta satisfaz todos os requisitos necessários para a criação da solução proposta. O web service possui três classes Java: PrevisaoCptec, Previsao e PrevisaoResultado. A primeira denominada "PrevisaoCptec" (Figura 6), é responsável pela acesso aos arquivos do CPTEC, criação do script e execução deste através do software GrADS. A outra classe denominada "Previsao" (Figura 7) através do método "getPrevisao" é responsável pela criação dos objetos, bem como alimentação das atributos destes e o controle da execução como um todo. Apenas o método denominado "getPrevisao" é publicado na WSDL do serviço. Para invocar este método é necessário informar os seguintes variáveis:

- Coordenadas Geográficas, latitude e longitude do ponto desejado;

- Data e hora da execução e geração dos arquivos do modelo numérico do CPTEC;

- Data e hora que se deseja obter as informações da previsão do tempo;

Após o desenvolvimento das classes Java, responsáveis pela execução do web service, foi realizada a publicação do serviço através do framework Axis. A confirmação da publicação pode ser visualizada na Figura 8 . 


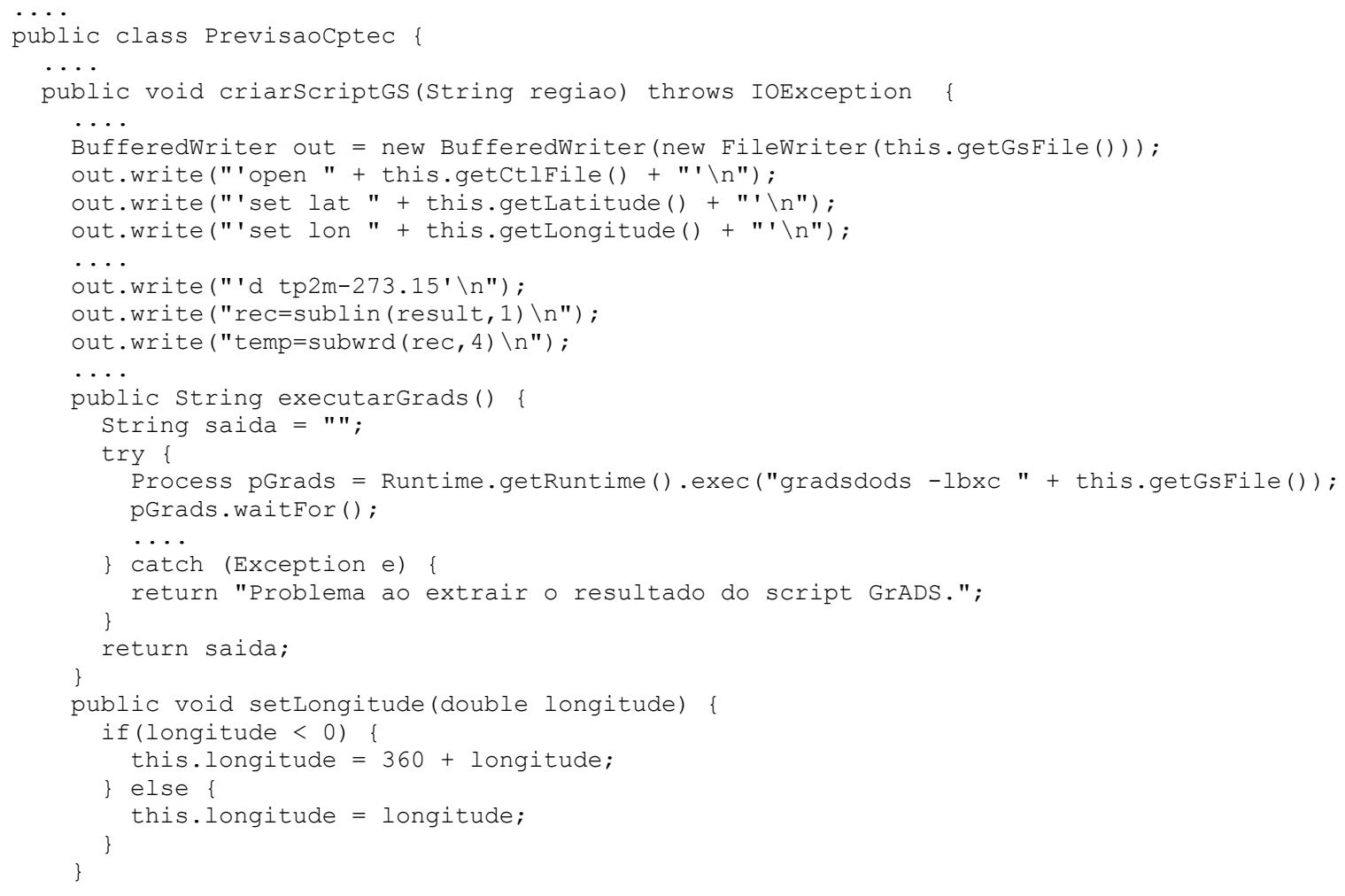

Figura 6. Partes do Código Fonte da Classe PrevisaoCptec.java

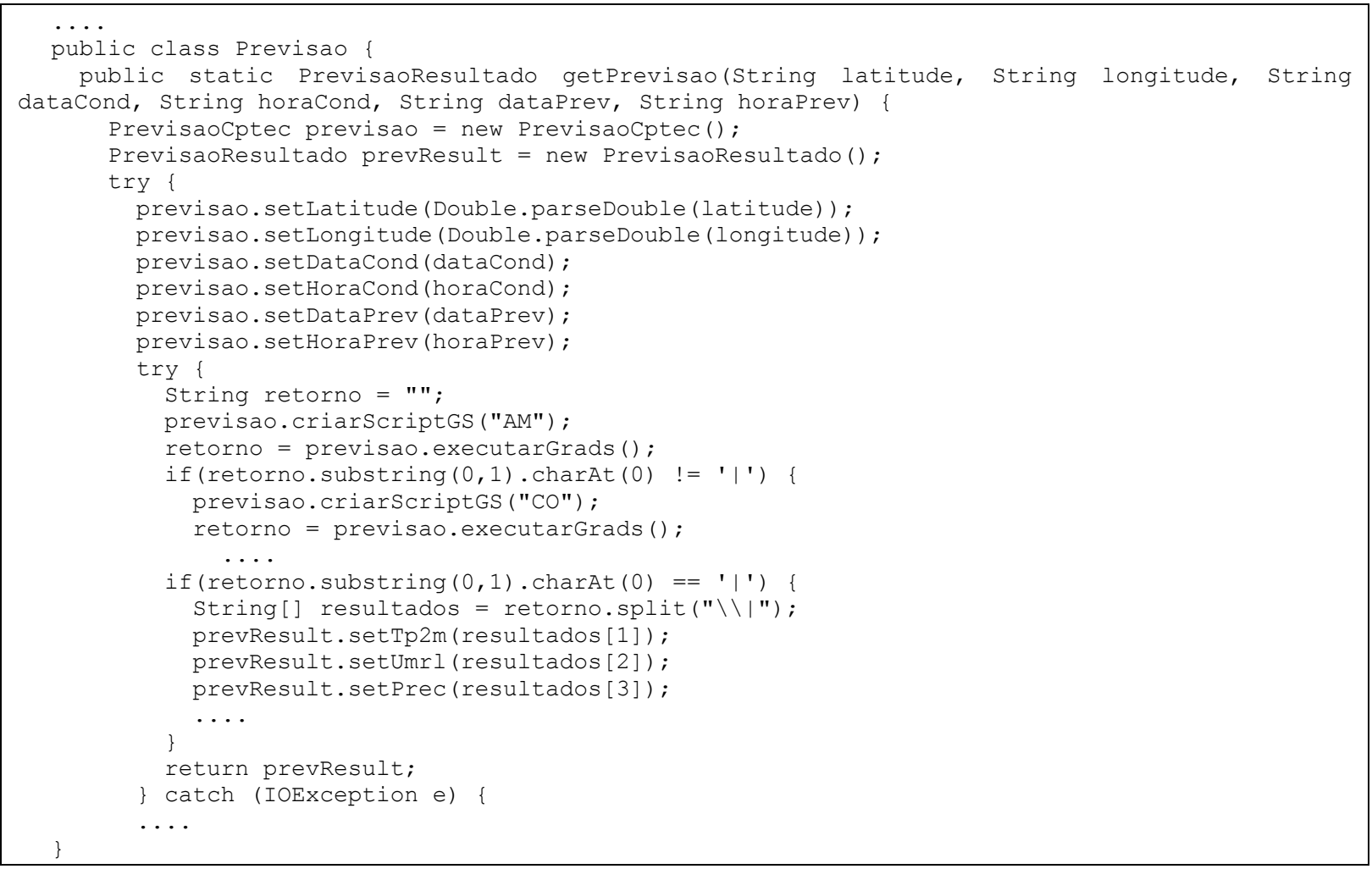

Figura 7. Partes do Código Fonte da Classe Previsao.java 


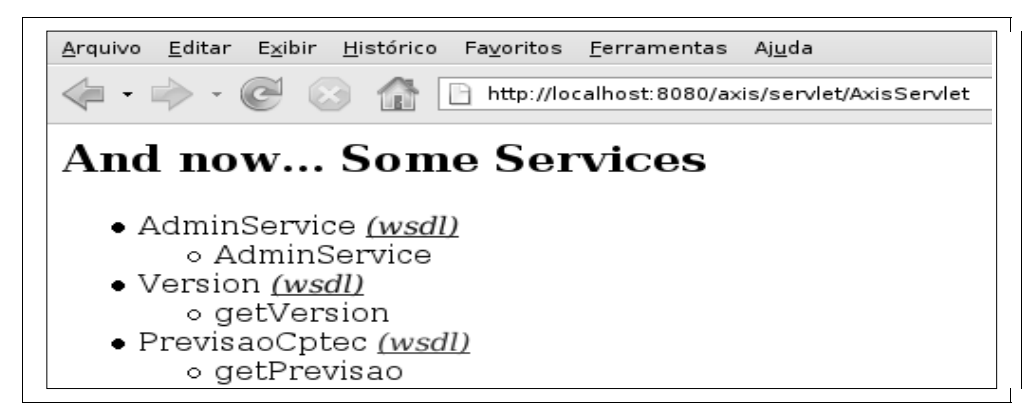

Figura 8. Relação dos serviços publicados

Acessando a WSDL do serviço publicado (Figura 9) é possível constatar o detalhamento deste, conforme mencionando anteriormente.

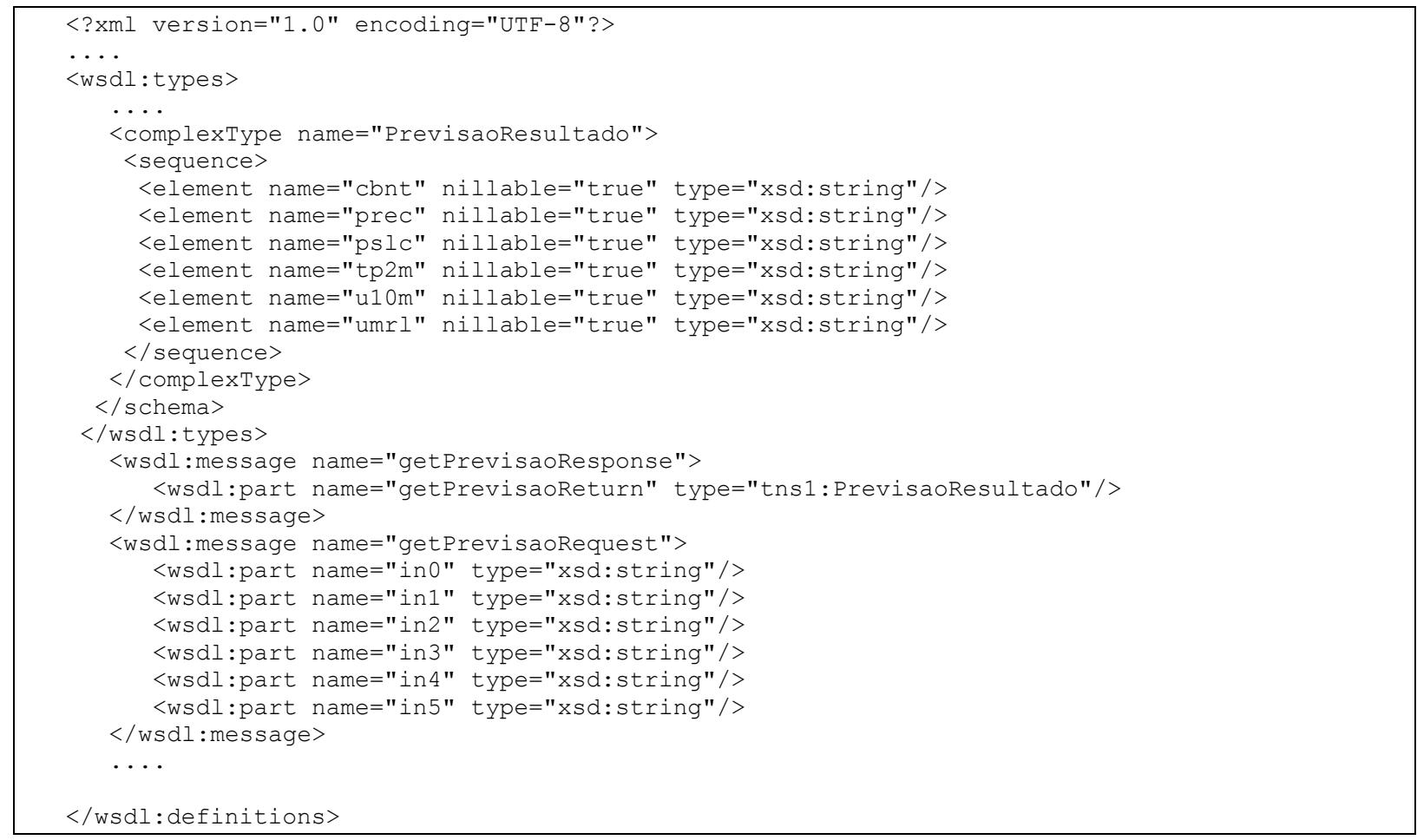

Figura 9. Partes da WSDL do Web Service PrevisaoCptec

\subsection{Detalhamento do processo de extração dos dados}

Conforme mencionando, diversos passos são executados dentro da classe "PrevisaoCptec" de forma que seja possível a consulta e extração dos dados dos arquivos do CPTEC, conforme detalhado a seguir:

1. Criação do objeto da classe PrevisaoCptec, validação e alimentação dos seus atributos, sendo necessário atentar-se ao parâmetro longitude, pois este pode ser informado com valores negativos. Neste caso é necessário somar 360 ao número negativo de forma que possamos ter a longitude de forma correta para o GrADS. A validação e formatação de datas também se fazem necessários.

2. Criação do script no formato GrADS, o qual possui uma sintaxe própria para seus scripts, portanto foi necessário criar um arquivo com a extensão ".gs", contendo os comandos deste software para posterior execução. 
3. Para executar o software GrADS foram utilizadas as classes Runtime e Process do Java, executando-o com o seguinte comando:

"gradsdods -lbxc nome_script.gs", onde:

a. o parâmetro "l" indica que o GrADS irá executar em modo landscape (paisagem);

b. o parâmetro "b" indica que será processado um arquivo no modo batch (lote);

c. o parâmetro "x" indica que ao finalizar a execução do script o GrADS deve encerrar o seu processo e liberar o shell do sistema operacional;

d. o parâmetro "c" utilizado para informar os comandos contidos no arquivo de script gerado anteriormente;

4. Validação dos dados de saída do GrADS.

5. Caso os dados do GrADS sejam retornados com sucesso é efetuada a criação do objeto PrevisaoResultado e alimentado os seus atributos.

6. Retorno do objeto PrevisaoResultado para o usuário/sistema requisitante;

Após a execução de todos os passos necessários para a consulta e extração das informações, o Web service retorna para o usuário/sistema solicitante um objeto do tipo "PrevisaoResultado" com os seguintes atributos: umidade relativa, nebulosidade, precipitação, temperatura, pressão ao nível do mar e velocidade do vento.

\subsection{Testes, protótipos e validações}

Após a confirmação da disponibilidade do serviço, foi realizado o primeiro teste através da chamada ao método getPrevisao, bem como a passagem dos parâmetros necessários, através da URL de um navegador web, conforme segue:

http://localhost: $8080 /$ axis/services/Previsaocptec?method=getPrevisao\&in0=-2.96\&in1=$59.93 \&$ in $2=20071024 \&$ in $3=12 \&$ in $4=20071028 \&$ in $5=06$

Resultado:

$<$ ?xml version="1.0" encoding="ISO-8859-1"?>

.

xmlns:ns1="Previsaocptec">

<cbnt xsi:type="xsd:string">0.374084</cbnt>

<prec xsi:type="xsd:string">0.00157213</prec $>$

<pslc xsi:type="xsd:string" $>1002.03</$ pslc $>$

<tp2m xsi:type="xsd:string" $>23.8405</$ tp2m>

<u10m xsi:type="xsd: string" $>0.645103</$ u10m>

<umrl xsi:type="xsd:string">75.9548</umrl>

$\cdots$

Figura 10. Endereço e partes do resultado da requisição para teste do Web Service

Com o objetivo de desenvolver um cliente completo para o web service desenvolvido, foi criada uma página Web utilizando HTML (Hypertext Markup Language), Javascript, bibliotecas do Google Maps, CSS (Cascading Style Sheet) e a linguagem de scripts PHP. O funcionamento do cliente desenvolvido pode ser compreendido através do fluxo apresentado na Figura 11. O cliente Web desenvolvido foi dividido em três partes:

- Página Web: Utiliza os recursos HTML e CSS para criação de páginas para a internet;

- Javascript: Utilizado para importação das bibliotecas do Google Maps, de forma a possibilitar a visualização e manipulação dos mapas. Através do Javascript foi possível utilizar técnicas do AJAX para fazer solicitações ao cliente do Web Service;

- PHP - Utilizado para o desenvolvimento do cliente do web service utilizando as biblioteca NuSOAP, conforme Figura 14. 


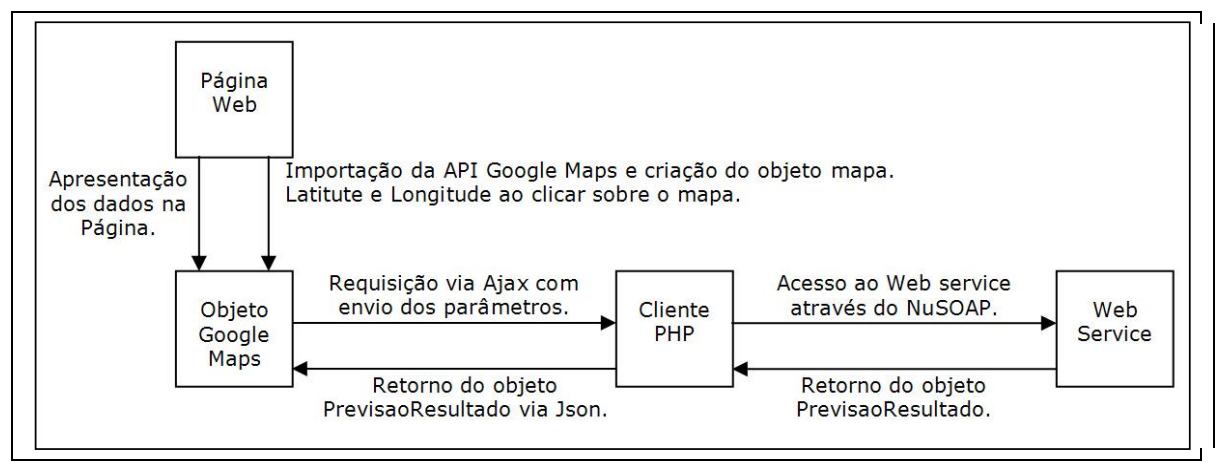

Figura 11. Fluxo e funcionamento do cliente $\mathrm{Web}$

<script src="http://maps.google.com/maps?file=api\&amp;v=2\&amp;key=ABQIAAAAjIsDGBlv" type="text/javascript"></script>

Figura 12. Linha executando a importação das bibliotecas do Google Maps

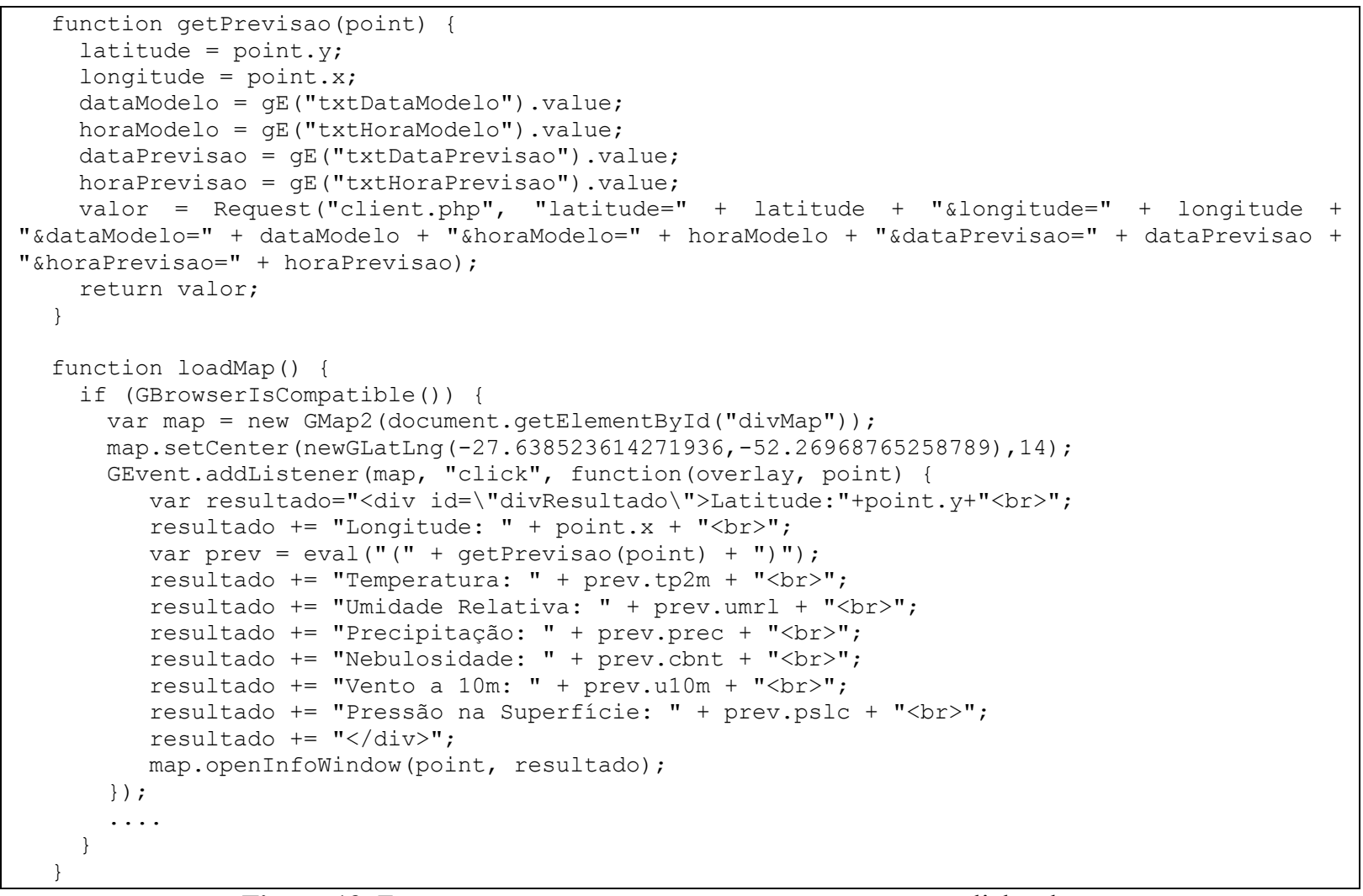

Figura 13. Funções para carregar o mapa e tratar o evento onclick sobre o mesmo

O resultado do cliente Web pode ser visualizado na Figura 15. Após selecionar a data e hora do modelo, bem como a data e hora da previsão desejada, clicado-se sobre um ponto no mapa (neste caso sobre a cidade de Manaus-AM). Ao clicar sobre o mapa é possível obter os pontos de latitude e longitude sobre o ponto clicado, sendo, após todos os parâmetros informados, enviados os dados ao cliente que realizou a solicitação. O resultado obtido do cliente PHP é apresentado em um "balão" sobre o ponto clicado no mapa. 


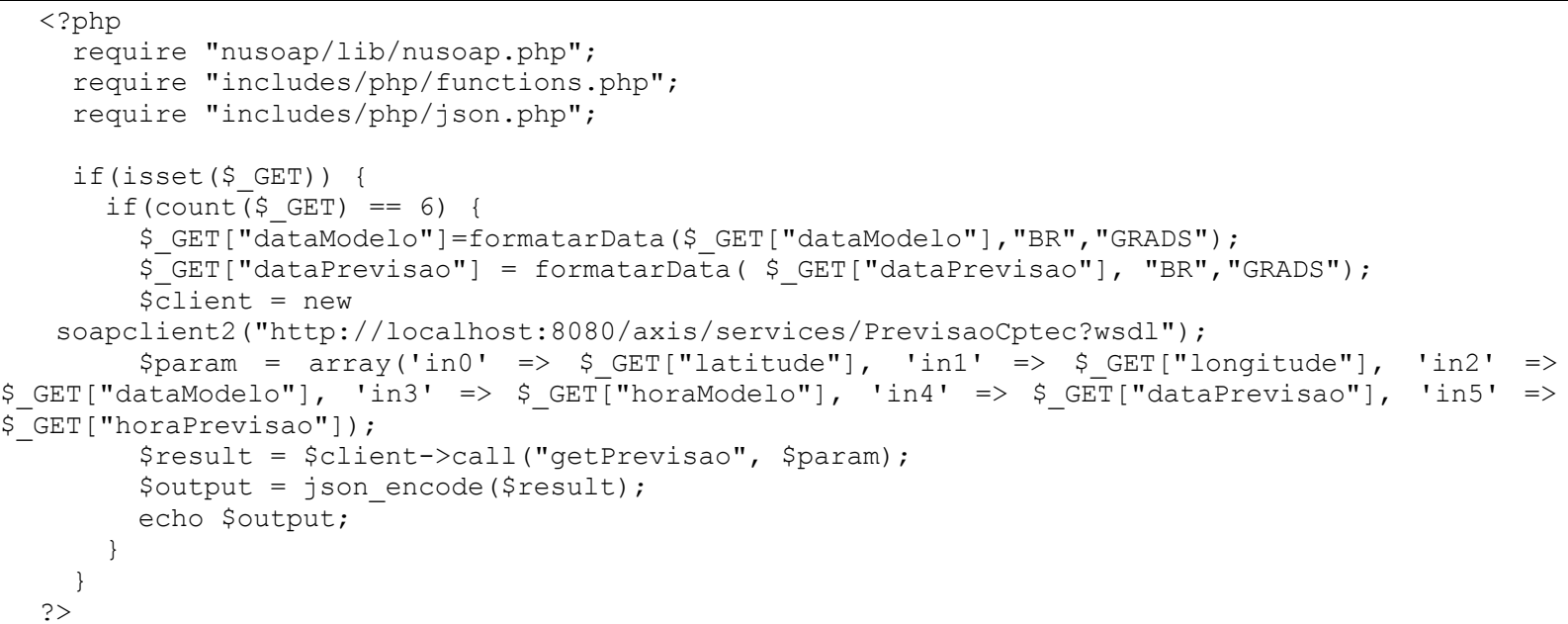

Figura 14. Código Fonte do cliente PHP

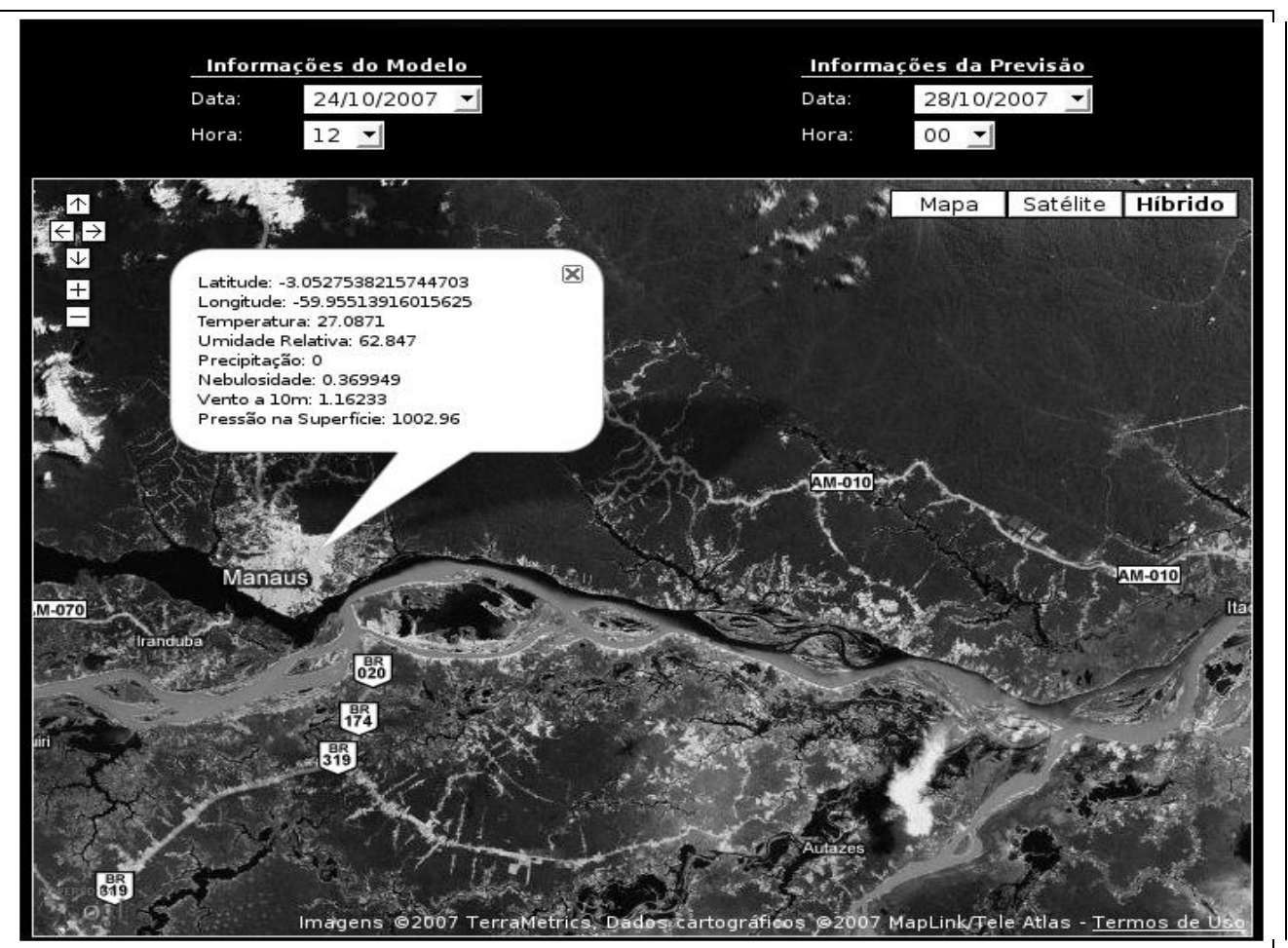

Figura 15. Página da internet como cliente do Web Service

\section{Discussão}

Embora a meteorologia seja uma ciência bem desenvolvida e os modelos numéricos complexos, gerando informações de previsão do tempo com altos índices de precisão [6,18], a distribuição dos dados georeferenciados é limitada a algumas pessoas que detém um conhecimento mais amplo sobre tecnologia, principalmente ao software GrADS, um dos softwares mais utilizados na área.

Além de um conhecimento aprimorado das tecnologias envolvidas para a extração das informações é necessária uma intervenção manual do usuário, de forma que este copie os arquivos disponibilizados pelo CPTEC e tratados através do software GrADS, tornando o processo de extração das informações custoso. 
Durante a pesquisa bibliográfica e o desenvolvimento do trabalho foi possível identificar diversos trabalhos correlatos abordando assuntos semelhantes. A diferença destes trabalhos para o apresentado é que os demais retornam informações em forma de gráfico ou imagens visuais, além de não trabalharem com dados georeferenciados e sim com nomes de cidades e localidades as quais se deseja obter a previsão de tempo.

Foram detectadas algumas dificuldades durante o processo de desenvolvimento do trabalho, principalmente com relação a obter informações sobre o funcionamento da ferramenta GrADS utilizada pelo CPTEC, bem como pessoas capacitadas que utilizem o software. Outra dificuldade encontrada foi com relação ao material e exemplos de utilização da ferramenta GrADS, bem como o formato em que os arquivos estavam disponibilizados. O GrADS possui uma sintaxe de programação própria para a extração das informações, o que exige um grande esforço para compreender alguns comandos e instruções do software.

Os arquivos disponibilizados pelo CPTEC com os dados das previsões de tempo e clima estão divididos em diretórios correspondentes a cada região brasileira. Mas como o Web Service apresentado trabalha com coordenadas georeferencias, encontrou-se dificuldade em descobrir qual a região corresponde a coordenada informada de forma que fosse possível procurar os dados nos diretórios corretos.

Com relação ao script para sincronismo dos dados com o CPTEC, não houve problemas e a linguagem de scripts PHP atendeu de forma completa os requisitos das tarefas programadas. Assim como o script de sincronismo, não houve problemas para a criação do Web Service desenvolvido com a linguagem Java, atendendo todas as expectativas com relação à solução proposta. O servidor de aplicações Tomcat, juntamente com o framework Axis, facilitou o processo de publicação e disponibilização do serviço.

Os testes e validações desenvolvidos ocorreram de acordo com o objetivo e propósito da solução, correspondendo as expectativas e facilidades que o Web Service vinha a propor.

\section{Conclusões}

A solução proposta alcançou os objetivos estabelecidos em sua plenitude, implementando-se uma estrutura computacional capaz de fornecer informações de previsão de tempo e clima, através de dados georeferenciados, de forma automática sem interferir na interoperabilidade da solução.

O desenvolvimento, os estudos e as tecnologias empregadas durante o desenvolvimento do trabalho atenderam de maneira satisfatória todos os quesitos em que as mesmas se propunham.

Os testes e as validações deixaram claras as funcionalidades, viabilidade e usabilidade do serviço. A solução mostra-se como uma ferramenta eficiente para usuários e/ou sistemas que necessitem de dados de previsões de tempo e clima através de dados georeferenciados.

A ferramenta apresentada disponibiliza fácil acesso e manipulação dos resultados obtidos, tendo em vista que trabalha com tecnologias padronizadas e consolidadas mundialmente, bem como sistemas e padrões baseados em software livre ou open-source, facilitando a integração e futuros aperfeiçoamentos desta.

\section{Trabalhos futuros}

Embora o trabalho aqui apresentado tenha atingido os objetivos propostos inicialmente, alguns pontos merecem atenção com o intuito de possibilitar a continuidade e o aperfeiçoamento da ferramenta desenvolvida. Como sugestão para trabalhos futuros destaca-se o desenvolvimento de uma funcionalidade na ferramenta que descubra uma região no Brasil com base nas coordenadas de latitude e longitude fornecidas fazendo com que o web service possa consultar os dados diretamente no diretório específico de cada região. Tendo em vista que a ferramenta desenvolvida retorna apenas seis indicadores de previsão de tempo e clima conforme apresentado no artigo, uma nova característica da ferramenta poderia ser a criação de funcionalidades que permita ao cliente informar quais dados de previsões ele deseja, obtendo estes de forma dinâmica na base de dados do projeto. 


\section{Referências}

[1] AXIS, W. Apache Axis Framework. Wikipédia - Enciclopédia Livre. Disponível em: $<$ http://pt.wikipedia.org/wiki/Apache_Axis>. Acesso em: out. 2010.

[2] BRANCO, R. A. Um estudo de caso sobre a sensibilidade do modelo ETA ao domínio e à resolução. Departamento de Meteorologia, UFRJ, 2007.

[3] CHAVES, R. R.; RODRIGUEZ, D. A. Apostila GrADS. Instituto Nacional de Pesquisas Espaciais, INPE, 2001.

[4] CHOICE ADMIN'S. Crontab Resource for Unix SysAdmins. Disponível em: $<$ http://www.adminschoice.com/docs/crontab.htm>. Acesso em: out. 2010.

[5] CPTEC. Modelo ETA. Centro de Previsões de Tempo e Estudos Climáticos. Disponível em: $<$ http://www.cptec.inpe.br/prevnum/exp_eta.shtml >. Acesso em: nov. 2010.

[6] FUNCEME. Tempo e Clima. Fundação Cearense de Meteorologia e Recursos Hídricos. Disponível em: $<$ http://www.funceme.br/DEMET/saiba_mais/saiba_mais.htm>. Acesso em: set. 2009.

[7] GONÇALVES, B.L. M. F. Estudo da concepção, definição e projeto de aplicações Web enriquecidas com Ajax. Universidade Federal do Mato Grosso, 2006.

[8] GRIMM, A.M. Meteorologia Básica - Introdução a Meteorologia. Disponível em: $<$ http://fisica.ufpr.br/grimm/aposmeteo>. Acesso em: nov. 2010.

[9] JAVA. Plataforma Java. Wikipédia - Enciclopédia Livre. Disponível em: $<$ http://pt.wikipedia.org/wiki/Plataforma_Java>. Acesso em: nov. 2010.

[10] MAPS. Google Maps. Wikipédia - Enciclopédia Livre. Disponível em: $<$ http://pt.wikipedia.org/wiki/Google_Maps>. Acesso em: out. 2010.

[11] PHP. Manual do PHP. PHP: Hypertext Preprocessor. Disponível em: $<$ http://www.php.net/manual/pt_BR/preface.php>. Acesso em: set. 2010.

[12] PROJECT APACHE. Web Services Axis. Disponível em: <http://ws.apache.org/axis>. Acesso em: out. 2010.

[13] QUADRO, M. Estrutura da Meteorologia. Curso Técnico de Meteorologia, Centro Federal de Educação Tecnológica de Santa Catarina, 2004.

[14] SUN MICROSYSTEMS. Web Services Overview. Web Services for the Java Platform. Disponível em: $<$ http://java.sun.com/webservices/>. Acesso em: ago. 2010.

[15] Tomcat, W. Apache Tomcat. Wikipédia - Enciclopédia Livre. Disponível em: $<$ http://pt.wikipedia.org/wiki/Apache_Tomcat>. Acesso em: out. 2010.

[16] TUBELIS, A.; NASCIMENTO, F. Meteorologia descritiva: Fundamentos e aplicações brasileiras. São Paulo: Nobel, 1980.

[17] UBUNTU-BR. Ubuntu-BR. Comunidade do Ubuntu no Brasil. Disponível em: < http://www.ubuntubr.org/>. Acesso em: ago. 2007.

[18] USP. Ciências Atmosféricas ou evolução da Meteorologia? Departamento de Ciências Atmosféricas Universidade de São Paulo (USP). <http://www.dca.iag.usp.br/www/cursos.php>. Acesso em: ago. 2010.

[19] WEB. Web Service. Wikipédia - Enciclopédia Livre. Disponível em: $<$ http://pt.wikipedia.org/wiki/Web_service>. Acesso em: out. 2010.

Revista Brasileira de Computação Aplicada (ISSN 2176-6649), Passo Fundo, v.3, n. 2, p. 2-16, set. 2011 